ERRATUM

doi:10.1038/nature15255

\title{
Erratum: Genetic diversity and evolutionary dynamics of Ebola virus in Sierra Leone
}

Yi-Gang Tong, Wei-Feng Shi, Di Liu, Jun Qian, Long Liang, Xiao-Chen Bo, Jun Liu, Hong-Guang Ren, Hang Fan, Ming Ni, Yang Sun, Yuan Jin, Yue Teng, Zhen Li, David Kargbo, Foday Dafae, Alex Kanu, Cheng-Chao Chen, Zhi-Heng Lan, Hui Jiang, Yang Luo, Hui-Jun Lu, Xiao-Guang Zhang, Fan Yang, Yi Hu, Yu-Xi Cao, Yong-Qiang Deng, Hao-Xiang Su, Yu Sun, Wen-Sen Liu, Zhuang Wang, Cheng-Yu Wang, Zhao-Yang Bu, Zhen-Dong Guo, Liu-Bo Zhang, Wei-Min Nie, Chang-Qing Bai, Chun-Hua Sun, Xiao-Ping An, Pei-Song Xu,

Xiang-Li-Lan Zhang, Yong Huang, Zhi-Qiang Mi, Dong Yu, Hong-Wu Yao, Yong Feng, Zhi-Ping Xia, Xue-Xing Zheng, Song-Tao Yang, Bing Lu, Jia-Fu Jiang, Brima Kargbo, $\mathrm{Fu}-\mathrm{Chu} \mathrm{He}$, George F. Gao, Wu-Chun Cao \& The China Mobile Laboratory Testing Team in Sierra Leone

Nature 524, 93-96 (2015); doi:10.1038/nature14490

This Letter should have contained an associated Creative Commons statement in the Author Information section. In addition, the Fig. 3c legend should have stated that the bar chart was adapted, with permission, from Ebola response roadmap - Situation report, Figure 3; http://www.who.int/csr/disease/ebola/situation-reports/en (accessed 1 April 2015) ${ }^{1}$. These issues have now both been corrected in the online versions of the paper.

1. World Health Organization. Ebola response roadmap - Situation report. http:// www.who.int/csr/disease/ebola/situation-reports/en (accessed 1 April 2015). 